\title{
Older people's experiences of falling and perceived risk of falls in the community: a narrative synthesis of qualitative research
}

\section{Abstract}

Background. Falls are a major problem for older people and healthcare services. Falling is generally recognised as an intrinsic risk with aging.

Aim. To examine qualitative research exploring older people’s experiences of falling and the perceived risk of falling in the the community.

Design. Narrative synthesis of qualitative literature as described by Popay et al. (2006). CINAHL, Medline, EMBASE, PsychInfo and BNI were searched 1999-2015.

Methods. Narrative synthesis. Concepts were analysed thematically, examining the experiences of older people in the community who have fallen or may be considered at risk of falling.

Results. Eleven papers fitted the inclusion criteria and revealed a series of themes. These were: falls as a threat to personal identity, falls as a threat to independence, falls as a threat to social interaction and carefulness as a protective strategy.

Conclusion. Many older people reject the label of 'at risk of falling' because of the perceived implication of dependency and incompetence. To be considered 'at risk' of falling is perceived as threatening the identity of individuals who are comfortable maintaining their own independence. However, there are also those who accept the risk of falling and in doing so choose carefulness as a personal strategy to manage the risk. For most older people, maintaining independence is the key motivator influencing their actions. Independence to pursue social interaction safeguards against loss of identity, social isolation and negative feelings of dependency.

Keywords. Older people, falls, community, qualitative methods, nursing, narrative synthesis. 


\section{Summary Statement of Implications for Practice}

What does this research add to existing knowledge in gerontology?

- Falls present problems for older people beyond physical harm, threatening their personal identity, independence and social interaction.

- Falls are negatively associated with ageing resulting in many older people denying their falls or risk of falling.

- The terminology of falls prevention is viewed negatively by older people, and may be the cause for rejecting these programmes.

- Despite multiple previous reviews on the topic, the problem persists meaning new interpretations are required.

What are the implications of this new knowledge for nursing care with older people?

- Many older people reject the label of 'at risk of falling' because of the perceived implication of dependency and incompetence. Management of falls risk needs to be portrayed more acceptably as constructive, proactive health behavior.

- The 'falls prevention' terminology used by healthcare professionals has an associated stigma. New terms to describe programmes to improve strength, balance and reduce risk should be employed.

How should the findings be used to influence practice or research?

- Understanding older people's reluctance to engage with falls prevention schemes should encourage nurses to consider alternative terminologies to inspire engagement. 
- Research is needed to understand the perception of falls in older people with dementia, but without capacity, living in the community.

- Nurses need to adopt a patient-centred approach by facilitating older people to make their own autonomous, informed decisions for self-management.

\section{Introduction}

Internationally falls are a major problem for older people and for health and social services. In the UK up to $30 \%$ of people aged over 65 fall each year, although only one in five falls require medical attention and less than one in ten results in a fracture (Gillespie et al. 2009). In a Global Report on Falls Prevention for the World Health Organisation, Yoshida (2007) reported that the frequency of falls increases with age and frailty; and most occur during the day, with only 20\% occurring at night. Men are more likely to fall while being active outdoors, while women are more likely to fall in the home. In fact, women are 40-60\% more likely to fall than men and over twice as much more likely to suffer fractures (Yoshida 2007).

Recent data on falls incidence are limited, though in the United Kingdom (UK), falls cost the National Health Service (NHS) more than $£ 2$ billion a year, and with the number of people over aged over 65 predicted to increase by 2 million by 2021, there are concerns that the trajectory is set to continue upwards (Tian et al. 2013). High income countries account for $25 \%$ of the total number of fatal falls worldwide, the highest rates occurring in European countries (Yoshida 2007). As a result, the UK National Health Service has initiated falls prevention programmes to manage the anticipated risk to people aged over 65 years of age. However, there is some evidence to suggest that efforts by healthcare professionals to intervene to reduce the risk of falls are poorly taken up and often rejected by the populations they are aiming to support (Yardley 2005; Nyman \& Ballinger 2007, Bun et al 2011, Bailey et al 2014). The geographical aspect may cause difficulties in some communities where the location of a group intervention may cause difficulties for older people willing to attend. Alternatively, falls prevention advice is regarded as useful in principle, but the common sense advice offered may not be perceived as relevant or appropriate (Child et al 2012, Yardley 2007).

While a large body of quantitative evidence exists including the causes, number and treatment of falls, there is rather less literature on the experiences and perspectives of older people themselves. Previous useful reviews of the qualitative evidence available to explore the experiences of older people likely to fall have also listed fatalism, denial under-estimation of the risk, poor health, poor functional ability, low expectations and the stigma associated with programmes that target older people as barriers to participation (Bunn 2011, Child et al 2012, Bailey et al 2014, McInnes et al 
2014). There is a need to address these barriers to falls prevention from a patient-centred standpoint and consider how nurses can assist patients manage their own risk. Thus, the focus of this review was to gain insight into the experience of older people who may fall in the community. The aim of the review was to examine qualitative research exploring older peoples lived experience, and perceived risk of falling in the community.

\section{Method}

Narrative synthesis of qualitative literature as described by Popay et al. (2006) was used to guide the development of this review. Popay's form of story-telling through narrative synthesis is an approach to the systematic review and synthesis of findings from multiple studies. It relies on the use of words and text to summarise and explain the findings of synthesis. The data was collected in narrative form and examined for conceptual overlap that occurred across the literature. In this way a process of translation of primary concepts were reported across the studies to explore similarities and differences between them (Popay et al 2006).

A systematic search of data-based literature using CINAHL, EMBASE, Medline, PsychInfo and BNI databases was undertaken using key search terms; frail older or elder, accidental falls or falls or falling, experience or perception and qualitative. Papers were included if they were written in English, published between 1999 to 2015, and included older people's perspectives of falls or falling in the community. Articles excluded were those not of a qualitative research design or those that were irrelevant to the research objective and were discarded if they were duplicated across databases, in a language other than English, opinions pieces or systematic literature reviews. Studies were excluded if they took place in hospitals or other care settings, if they were investigating the impact of a specific intervention or prevention programme, or if they were evaluating assessment tools. The search process was conducted by the first author and audited by a health librarian. Eleven papers were examined to evaluate rigour, credibility and relevance, using the Critical Appraisal Programme (CASP 2014) for qualitative research. Each paper was reviewed to determine the perceptions of older people towards falling and themes central to the perceptions of falling and risk as given by the informants. Thematic analysis was conducted by identifying the common behaviour and experience within the articles.

\section{Results}

The initial search yielded 628 articles. Eleven papers were identified as fitting the inclusion criteria (see figure 1), though two were reports from the same data set (Roe 2008, 2009). 
Examination of the literature revealed that falls have multiple effects on those falling well beyond any immediate physical harms leading to new understandings. Complex sequelae were revealed. These were; falls as a threat to personal identity, falls as a threat to independence, falls as a threat to social interaction and carefulness as a protective strategy (see Table 3).

These themes will be discussed in detail below.

\section{Falls as a threat to personal identity}

Falling affected confidence so severely that it represented a threat to personal identity. Acceptance of being at risk undermined an individuals' status as competent and independent and assumed an acceptance of becoming old and infirm. The importance of identity is the focus of two of the articles examined in this review in response to the evidence that some qualitative research identified older people distancing themselves from being labelled as 'at risk of falling' or as 'fallers'. Falling is a threat to the identity of a person who would prefer to be regarded as the type of person who does not fall (Dollard et al. 2012). It has been noted in the literature that one of the reasons offered for the poor uptake of falls prevention programmes is the concept that individuals reject the idea of being 'at risk' (Yardley et al 2006, Walker et al 2011, Dollard et al 2012).

In a relatively large study of 66 people, aged 61-94 years living in the community, it was found informants distanced themselves from the perceived threat to their identity because they did not wish to be considered old and infirm. Although falls prevention advice was considered useful, informants stated it was for 'other people in need of it' while they defined themselves a 'nonfallers' (Yardley, 2006). Their own falls were credited with being isolated events of carelessness or illness. A male informant, aged 81, had fallen out of bed four times in the previous 8 months and yet considered himself a 'non-faller'. However, study authors surmised that most people in the study were aware of their likelihood of falling, they simply refused to allow themselves to be defined as potential 'fallers’ (Yardley, 2006).These findings are congruent with subsequent similar studies.

In a study to explore the meaning of falling for older people, Walker et al (2011) found participants endeavoured to repudiate the label of an older person, as utilised by health services, i.e. everyone over 65. Their study included 11 informants who had participated in a falls prevention programme. The informants described a typical 'faller' as being over the age of 80 , with ill health and/or immobility. Only two of the nine informants considered themselves to be 'fallers'; one of whom had experienced five falls in an 18-month period. Study findings suggest that participants assessed themselves based on individual perceptions of health and role in society, and suggested a lack of recognition of age and gender difference might contribute to the low uptake of falls prevention 
services (Walker, Wendy, Porock Davina, Timmons 2011; Horton 2007). Health professionals should be aware that falls prevention messages are likely to be rejected if the target group associate the message with a negative identity (Dollard et al. 2012).

A few informants stated they had forgotten or simply denied their fall to the interviewer in Kong et al's (2002) study, which aimed to explore the psychosocial consequences of falling. The study included 20 older Chinese people who had recently experienced a fall, either in the community or in the hospital setting; half of whom had a previous history of falls prior to the most recent. This response was attributed to a defensive mechanism in order allow the individual to continue to view themselves as intact.

In a grounded theory study, involving 20 men and 20 women, Horton (2007) explored the influence of gender on older people's perception of their risk of falling and their actions to prevent future falls. All had experienced two or more falls in the previous 12 months and were living in the community. The differing perceptions of men and women influenced their actions to prevent further falls. Older men perceived themselves as 'responsible' and 'rational' individuals who expected to reduce their own risk of falling while older women's expectations of themselves and their peers explained their tendency to blame themselves or others for their fall (Horton 2007).

\section{Falls as a threat to independence}

Independence is important, the lengths individuals will go to maintain their independence says much about the individual managing their risk of falling. Independence is closely guarded and for some asking for assistance may be seen as an unacceptable loss of independence (Yardley et al 2006). Dependency means that an individual has to conform to other people's goodwill, thus compromising their own needs. Older people may be unwilling to ask for assistance as this may be may be another step towards surrendering their independence. Individuals will try to maintain their independence as far as possible because a restriction in activity will lead led to feelings of frustration and a sense of loss related to being unable to do the things they could do before (Hawley 2009, Kong 2006). For some individuals there may also exist a sense of being a burden to families, and being unable to fulfil their usual role expectations at home (Kong et al. 2002).

\section{Falls as a threat to social interaction}

For the individual who falls, social interaction can be jeopardised by restricting activity, reducing the ability to leave home and leading to isolation which in turn, results in feelings of frustration and anxiety (Roe 2009). 
Social interaction is important for a good quality of life. Walker et al (2011) emphasised the need for social interaction, because it naturally places individuals into groups and categories from which a sense of identity can develop. Hawley reported one participant who described losing her main chance for social interaction because she could no longer go to church, and this had clearly impacted on her quality of life (Hawley 2009). Formal care and informal support networks can provide support that is highly valued at a time when an individual is vulnerable due to unexpected changes in their living circumstances. Older people, most often women, living alone with the risk of falling derive support from social meetings where, combined with physical activities, they can challenge the limits of their own abilities (Berlin Hallrup et al. 2009; Roe et al. 2009; Hawley 2009). In view of this, it is sensible to consider social interaction an important component of any falls prevention programme.

Social interactions are not only important to individuals for peer support, they also represent a support network for health professionals in their endeavours to encourage individuals to engage in programmes to increase and improve mobility. Hawley (2009) revealed that relationships with professionals, families and friends as part of a social network during and after falls rehabilitation can impact on uptake and the continuation of exercise (Hawley 2009). For one of the female participants in this study her only social interaction was during the rehabilitation programme, and this provided the encouragement she needed to retain her independence. For the health professional, providing an individualised programme and follow up helps to engage the individual by providing another benefit; personal interaction. As if to demonstrate the power of health professionals in influencing participation, Walker (2011) found that many participants attended and continued with a programme out of respect for those who referred them or delivered it, as opposed to the feeling they needed the service or may benefit. In Hawley's study, some individuals stated that unless they had someone to tell them to follow their exercises they didn't bother the continuation of home exercise. Indeed, if these activities are characterised by fun and games, in a trust based atmosphere, there are many potential health benefits, including improvements to mobility (Berlin Hallrup et al. 2009).

Local informal care and support networks are as important as formal care for older people at risk of falls or who have fallen (Roe et al. 2009). A different perspective comes from the person living at home alone, because of the challenges in maintaining the range of personal choices around their living arrangements. Good relationships with professional helpers must be ensured to ensure help where it is necessary (Mahler \& Sarvimäki 2012). 


\section{Carefulness as a protective strategy}

Carefulness as a protective strategy is a theme throughout the literature reported here. Moving more carefully is safer, and even if there is a reduction in living space, mobility can be maintained (Berlin Hallrup et al. 2009). Regardless of whether an individual acknowledges a fear of falling, most informants recognised the need for carefulness. Being careful in response to possible risk could be considered in a positive light, since carelessness was often criticised. Indeed, women would accept blame if they could attribute their fall to carelessness. Some older people chastised and blamed themselves for not taking care when they fell, accepted responsibility for their behaviour as the cause of their fall, and claimed to take more care to prevent further falls (Dollard et al. 2012). In this way they minimised the perception that they were the type of person who could fall. This helped them to maintain their identity as an independent person.

As previously noted, gender differences were evident in the literature. Although men and women had largely differing approaches to the risk of falling, both employed a careful approach to managing the possibility. Taking care was regarded positively by both men and women, despite the differing perceptions of risk-taking behaviour. Men were explicit in their 'rational' approach to the risk of falling, however when women fell, they were inclined to blame self-blame, exerting their own sense of control by implying it was their decision to place themselves in the position of risk (Horton 2007).

In two studies exclusive to older women, Berlin Hallrup et al (2009) and Mahler and Sarvimaki (2011) utilised a phenomenological approach to illuminate the experiences of older women living alone with the risk of falling. These papers suggest that having the awareness of increased risk and often the fear of falling, too much 'carefulness' could result in women living alone to become isolated as they restricted themselves to their familiar surroundings. As a result, it became more difficult for the women to maintain social relationships meaning they became increasingly isolated (Berlin Hallrup et al. 2009). However, the women used their own individual strategies to find a balance between the risk of injury and mobility and so the degree to which each employed carefulness differed according to the individual (Berlin Hallrup et al. 2009; Mahler \& Sarvimäki 2012).

The literature suggests that older people reflected on their falls and acknowledged it in one of two ways; either they acknowledged their fear, faced it and took steps to minimize it by being careful; or, alternatively, they chose to accept the risk of falling but would choose not to be worried by this risk (Roe et al 2008). Even with the latter option however, they would be careful to avoid what risks they can (Roe et al. 2008). Health professionals describe the fear of falling as a restriction of 
activity however, for the older people, the fear of falling mainly constituted adaptation and finding a balance in their daily lives (Mahler \& Sarvimäki 2012).

\section{Discussion}

When viewed as an entire discourse analysis reveals new insights into the issues of falling in older people.

Falls are perceived as a consequence of ageing and part of the life course. Generally, older people are aware that the likelihood of falling increases with age. Falls prevention programmes, especially those incorporating strength and balance exercises, can significantly reduce the risk of falling (Child et al 2012, Yardley 2006). However, uptake rates for these programmes can be as low as $50 \%$, sometimes as low as $10 \%$, and without high participation rates interventions are unable to prove their effectiveness within the population (Yardley et al 2006).

The evidence from the literature provides the overarching impression that many older people believe falls prevention advice is simply common-sense, and therefore irrelevant and of little use. This denial of personal relevance illustrates the difficulty health professionals are likely to experience as they work towards persuading older people to undertake physical exercise to prevent or rehabilitate following a fall. Not all people aged over 65 accept the immediacy of the need to protect themselves against falling. Falls may be identified as a lack of control, which for many older people, is unpalatable. However, what is becoming clear through the literature is that there are levels of fitness in people over 65, mainly between those who are physically able and those who are not. The literature reviewed suggests that those who are physically able reject what they consider patronising, common-sense advice from healthcare professionals to be careful. Those who may not be able often choose to deny their deterioration and instead aspire to what they have been able to do previously. They do not wish to be considered at risk or placed in a category of 'older people' as this threatens their identity as active, independent people.

However, there are older individuals who have accepted that their bodies are ageing, therefore may be less able, and learn to manage the risk of falling through adopting carefulness strategies. Even those individuals who refused to recognise themselves at risk were prepared to accept that common-sense advice is to be careful, and this was the primary strategy employed by those who did consider themselves at risk of falling. However, it is when carefulness morphs into a fear of falling it becomes debilitating to the individuals' independence. An exaggerated adaptation of the risk of falling and an aging body can increase the possibility of an older person becoming socially isolated in their own home (Berlin Hallrup et al. 2009). The challenge for health professionals is to 
encourage the positive outcomes of carefulness without sanctioning over-vigilance to the extent that it becomes limiting.

Maintaining independence is a crucial motivator for individuals' decisions in how they approach falls. Either an individual does not wish to be considered without independence or they are striving for it. It is their attitude towards their independence that defines their approach to the risk of falling. The concept of mobility is a common denominator when individuals were asked to define people who fall (reference needed). It is generally accepted that anyone from any age group could fall, however the older an individual the higher the risk of falling because it is accepted that strength and balance deteriorate with age. Herein lies the rub; people are prepared to accept that there is a risk to themselves, that some choices mean greater risk than others, or even accept they make their own risky choices. However, once there is a suggestion their choices may represent an acceptance of old age, and all that goes with it.

The fact social interaction is excluded from Mahler and Sarvimaki's (2011) study is telling. The study included five community-dwelling women over 80 talking about their perspectives on daily life. The fear of falling dominated the lives of the women, their individual autonomy was maintained against a backdrop of social isolation and dependence on caring staff. These findings are an echo of Porter's (2008) earlier study exploring the experiences of frail older women who had fallen at home alone. Indeed, Roe et al (2008) described feeling alone as a feature of falls, where most falls were reported when women were living alone. This is an issue addressed by in their later article where they concluded that local informal care and support networks are as important as formal care for older people at risk of falls (Roe et al. 2009). Social interaction was identified as playing a crucial role in falls prevention as it enables the person who has fallen to share experience, gain assistance and encouragement. The importance of positive interaction cannot be over-stated, as Walker (2011) elucidates, the difficulty is when health professionals fail to recognise the need to personalise the interaction and put the individual at the centre of the discussion and decision making. Using the benefits of attending a programme; focused on keeping fit, active, socialising - could be used to promote independent healthy living instead of the perceived inevitability of ageing and losing independence as feared by many people.

It is noteworthy that none of the papers address the issues of falling in people with dementia. Considerable difficulties arise with people who no longer have the capacity to comprehend risk or instruction and yet have the physical abilities to move freely. None of the articles discussed here refer to screening for cognitive impairment. This is significant because people with Alzheimers disease have twice the risk of falling as people the same age without the disease (Yoshida 2007). Only one paper addressed cultural issues (Kong et al. 2002), although this was produced outside of the UK, so current research presents a culture of homogeneity. This also needs to be addressed 
given the rising number of people with dementia in Black Minority and Ethnic communities. The Black Minority and Ethnic population in the UK and other Western countries is younger than the majority population and tends to have a higher proportion of younger onset dementia, so the overall burden of dementia is substantial (Mukadam 2015). The fact that these groups often present late to services suggests that there is much to be done by health professionals to encourage engagement with the services.

If we assume a patient-centred approach is the way forward, empowerment is fundamental. To manage this successfully nurses should not assume responsibility for telling people how to manage their risk of falling. Empowerment is based on meeting patients' needs; it is about assisting patients to get what they feel they need in order for them to make autonomous, informed decisions about their self-management. It has been indicated previously that promoting and supporting exercise as a constructive means of falls prevention can improve the service provided to people considered to be at risk of falling (Yardley et al 2006). Thus, healthcare professionals should negotiate choices for intervention in recognition that some individuals prefer to drive the decision-making process (McInnes 2011). However, nowhere has it been suggested taking the therapeutic relationship closer to patient-centredness. The empowerment approach involves facilitating and supporting patients to reflect on their own experience (Anderson and Funnel 2011). Self-reflection often leads to enhanced awareness and understanding of the consequences of self- management decisions - the role of the healthcare professional serving as a facilitator and expert resource.

\section{Limitations}

This review is limited in that it is restricted to papers published in English. Another limitation is the reasons for individuals not engaging with falls prevention programmes is never fully explored, this may be due to the fact research examining the effectiveness of a particular programme has been excluded from the literature search.

\section{Conclusion}

It is possible that management of falls risk could be portrayed more acceptably as a constructive, pro-active behaviour to maintain health, rather than as a purely defensive activity. Accepting the idea that we are all at risk of falling is an important consideration for health professionals in their approach to falls prevention.

Health professionals need to demonstrate they understand the issues faced by those who fall. Professionals with a different contextual understanding from individuals considered at risk of 
falling potentially contribute to the lack of engagement with falls prevention programmes.

Upholding identity through an individual approach, knowing an individual's approach to perceived risk through carefulness, maintaining lines of communication between individuals, reducing the possibility of social isolation and allowing them to share their experience are all ways that health professionals can demonstrate a patient-centred approach.

We need to understand the older people's reluctance to engage with falls preventions schemes in order for them to succeed but also to encourage nurses to consider what alternative strategies need to be employed to engage this population. It is possible that by forcing older people to face their vulnerabilities health professionals are acting counter-intuitively as current fall prevention activity appears to discredit the abilities of this group of people.

\section{References}

Anderson, A. Funnell, M. (2011) Patient Empowerment: Myths and Misconceptions. Patient Education Counsel, 79(3), pp.277-282.

Bailey, C. Jones, A. Goodall, D (2014) Falling across the life course: a qualitative evidence synthesis of lived experiences. Journal of Disability and Health, 7(3), pp.273-284.

Berlin Hallrup, L. et al., 2009. Elderly women's experiences of living with fall risk in a fragile body: a reflective lifeworld approach. Health \& Social Care in the Community, 17(4), pp.379-387. Available at: http://doi.wiley.com/10.1111/j.1365-2524.2008.00836.x.

Bun, F. Dickinson, A. Barnett-Page, E. McInnes, E. Horton. K, 2008. A systematic review of older people's perceptions of facilitators and barriers to participation in falls-prevention interventions. Ageing and Society, 28(4), pp.449-472

Child, S. Goodwin, V. Garside, R. Jones-Hughes, T. Boddy, K. and Stein, K. 2012. Factors influencing the implementation of fall-prevention programmes: a systematic review and synthesis of qualitative studies. Implementation Science, 7(1) pp.91-105

Critical Appraisal Skills Programme (CASP) 2014. CASP Checklists

(http://media.wix.com/ugd/dded87_29c5b002d99342f788c6ac670e49f274.pdf) Oxford. CASP

Dollard, J. et al., 2012. Falls in old age: a threat to identity. Journal of Clinical Nursing, 21(17-18), pp.2617-2625. Available at: http://doi.wiley.com/10.1111/j.1365-2702.2011.03990.x.

Gillespie, L.D. et al., 2009. Interventions for preventing falls in older people living in the community. Cochrane database of systematic reviews (Online), (2):CD0071(2), p.CD007146.

Hawley, H., 2009. Older adults’ perspectives on home exercise after falls rehabilitation: Understanding the importance of promoting healthy, active ageing. Health Education Journal, 
68(3), pp.207-218.

Horton, K., 2007. Gender and the risk of falling: a sociological approach. Journal of advanced nursing, 57(1), pp.69-76. Available at: http://www.ncbi.nlm.nih.gov/pubmed/17184375.

Kong, K.S.W. et al., 2002. Psychosocial consequences of falling: the perspective of older Hong Kong Chinese who had experienced recent falls. Journal of advanced nursing, 37(3), pp.23442. Available at: http://www.ncbi.nlm.nih.gov/pubmed/11851793.

Mahler, M. \& Sarvimäki, A., 2012. Fear of falling from a daily life perspective; narratives from later life. Scandinavian Journal of Caring Sciences, 26(14), pp.38-44.

McInnes, E. Seers, K. Tutton, L., 2011. Older people's views in relation to risk of falling and need for intervention: a meta-ethnography. Journal of Advanced Nursing 67(12), pp. 2525-2536.

Montbriand, M. 2004. Seniors' survival trajectories and the illness connection. Qualitative Health Research. 14(4), pp.449-461.

Mukadam, N. Waugh, A. Cooper, C. Livingston, G. 2015. What would encourage help-seeking for memroy problems among UK-based South Asians? A qualitative study. BMJ open, 5(9), p.e007990.

Nyman, S. \& Ballinger, C., 2007. Falls Prevention in Practice. Available at: http://www.helptheaged.org.uk/NR/rdonlyres/8D4F8B43-05EE-4B8B-97A1DADCA894894E/0/falls_lit_review_101207.pdf.

Popay, J. et al., 2006. Guidance on the Conduct of Narrative Synthesis in Systematic Reviews A Product from the ESRC Methods Programme. , (April 2006), pp.1-92. Available at: file:///C:/Users/Shazza/AppData/Local/Mendeley Ltd./Mendeley Desktop/Downloaded/Roberts et al. - Unknown - Guidance on the Conduct of Narrative Synthesis in Systematic Reviews A Product from the ESRC Methods Programm.pdf.

Roe, B. et al., 2009. Older people and falls: health status, quality of life, lifestyle, care networks, prevention and views on service use following a recent fall. Journal of Clinical Nursing, 18(16), pp.2261-2272. Available at: http://doi.wiley.com/10.1111/j.1365-2702.2008.02747.x.

Roe, B. et al., 2008. Older people’s experience of falls: Understanding, interpretation and autonomy. Journal of Advanced Nursing, 63(6), pp.586-596.

Tian, Y. et al., 2013. Exploring the system-wide costs of falls in older people in Torbay. London: The King's Fund, pp.1-12. Available at: http://www.kingsfund.org.uk/sites/files/kf/field/field_publication_file/exploring-system-widecosts-of-falls-in-torbay-kingsfund-aug13.pdf.

Walker, Wendy, Porock Davina, Timmons, S., 2011. The importance of identity in falls prevention individual should promote better engagement in intervention. Nursing Older People, 23(2), pp.21-26.

Yardley, L., 2005. Older people’s views of advice about falls prevention: a qualitative study. 
Health Education Research, 21(4), pp.508-517. Available at:

http://www.her.oxfordjournals.org/cgi/doi/10.1093/her/cyh077.

Yoshida, S., 2007. A Global Report on Falls Prevention Epidemiology of Falls. WHO report, pp.140. 